\title{
Red-Brown Material
}

National Cancer Institute

\section{Source}

National Cancer Institute. Red-Brown Material. NCI Thesaurus. Code C124515.

Matter that is red and brown in color. 